\title{
WEAK IDEAL INVARIANCE AND ORDERS IN ARTINIAN RINGS: CORRIGENDUM
}

\author{
John A, BeACHY
}

Let $R$ be an associative ring with identity element which has Krull dimension on the left (see [3] for the relevant definitions). For any ordinal $\alpha$, and any module $R^{X}$, let $\tau_{\alpha}(X)$ denote the sum in. $X$ of all cyclic submodules of Krull dimension less than $\alpha$. (The Krull dimension of a module $X$ will be denoted by $|X|$.) It is clear that if $X^{\prime}$ is a submodule of $X$, then $\tau_{\alpha}\left(X^{\prime}\right)=X^{\prime} \cap \tau_{\alpha}(X)$ and that $f\left(\tau_{\alpha}(X)\right) \subseteq \tau_{\alpha}(Y)$ for any $R$-homomorphism $f: X \rightarrow Y$. Thus $\tau_{\alpha}$ defines a torsion preradical.

It has been pointed out to the author by Professor Ann Boyle that in [1] the torsion preradical $\tau_{\alpha}$ was incorrectly assumed to always determine a torsion radical. Difficulties may arise if $\alpha$ is a limit ordinal and $R$ is not left Noetherian. The main result of [1], Theorem 7, is valid as stated. The necessary corrections to the proof can be made easily by using the proposition proved in this note. The statements of Theorem 4 and Theorem 5 of [1] can be corrected by making the additional assumption that $\tau_{\alpha}$ is a torsion radical. A revised version of Theorem 6 is given below.

To show that $\tau_{\alpha}$ is a torsion radical, it is necessary to show that $\tau_{\alpha}\left(X / \tau_{\alpha}(X)\right)=(0)$ for all modules $R^{X}$. It is sufficient to show that if $A$ and $B$ are left ideals with $A \subseteq B, \tau_{\alpha}(B / A)=B / A$ and $|R / B|<\alpha$, then $|R / A|<\alpha$. This follows immediately if $R$ is left Noetherian. If $\alpha$ is not a limit ordinal, then $|B / A|<\alpha$ by Corollary 4.2 of [3], and so

Received 30 October 1981. 
$|R / A|<\alpha$

Definitions and notation are those of [1], and the following notation will be fixed throughout. The prime radical of $R$ will be denoted by $N$, and the Krull dimension of $R$ will be denoted by $\alpha$. The intersection of all prime ideals $P$ of $R$ such that $|R / P|=\alpha$ will be denoted by $N_{\alpha}$.

PROPOSITION. (a) If $N$ is left weakly ideal invariant, then $\tau_{\alpha}$ is a torsion radical.

(b) $N$ is left weakly ideal invariant if and only if $N_{\alpha}$ is left weakly ideal invariant.

(c) If $R / N$ is $K$-homogeneous and $C(N) \subseteq C(0)$, then $N$ is left weakly ideal invariant.

Proof. (a) Let $C=\{c \in R|| R / R c \mid<\alpha\}$. By Corollary 5 of [4], if $D$ is a left ideal of $R$ with $|R / D|<\alpha$, then $D \cap C \neq \varnothing$. The proof that $C$ is a left Ore set is similar to the first part of the proof of Theorem 8 of [4]. (Given $c \in C$ and $a \in R$, let $A=\{r \in R \mid r a \in R c\}$. Then $|R / A|<\alpha$, and so there exists $c^{\prime} \in A \cap C$, which implies that $c^{\prime} a=a^{\prime} c$ for some $a^{\prime} \in R$. Furthermore, if $c, d \in C$, then $c d \in C$ since $|R / R c d|=\sup \{|R / R d|,|R d / R c d|\}$ and $|R d / R c d| \leq|R / R c|$.$) Thus the filter of left ideals$ $\{D \subseteq R \quad|R / D|<\alpha\}$ which defines $\tau_{\alpha}$ has as a basis the set $\{R c \mid c \in C\}$, and since $C$ is a left ore set, it follows immediately that $\tau_{\alpha}$ is a torsion radical.

(b) Let $I$ be the intersection of all minimal prime ideals $P$ of $R$ with $|R / P|<\alpha$ (if this intersection is empty, then of course $N=N_{\alpha}$ ) and let $D$ be a left ideal of $R$ with $|R / D|<\alpha$. Then $|R / I|<\alpha$ and $I N_{\alpha} \subseteq I \cap N_{\alpha}=N$, so $N_{\alpha} / N$ and $N_{\alpha} D / N D$ are left $R / I$-modules, which forces $\left|N_{\alpha} / N\right|<\alpha$ and $\left|N_{\alpha} D / N D\right|<\alpha$. Since

$$
|N / N D| \leq\left|N_{\alpha} / N D\right|=\sup \left\{\left|N_{\alpha} D / N D\right|,\left|N_{\alpha} / N_{\alpha} D\right|\right\}
$$

and

$$
\left|N_{\alpha} / N_{\alpha} D\right| \leq\left|N_{\alpha} / N D\right|=\sup \left\{\left|N_{\alpha} / N\right|,|N / N D|\right\}
$$


it follows that $|N / N D|<\alpha$ if and only if $\left|N_{\alpha} / N_{\alpha} D\right|<\alpha$.

(c) Let $D$ be a left ideal of $R$ with $|R / D|<\alpha$. Then $\operatorname{Hom}(R / D, E(R / N))=(0)$ since $R / N$ is $K$-homogeneous, and so there exists $d \in D \cap C(N)$. If $|R / R d|=B$, then $\beta<\alpha$ since by assumption $C(N) \subseteq C(0)$ and hence $d$ is a regular element of $R$. For any element $x \in N \cap D$, if $r x=a d$ for $r, a \in R$, then $a d \in N$ implies that $a \in N$ since $d \in C(N)$, and so $r x \in N d$. Thus

$$
\{r \in R \mid r x \in N d\}=\{r \in R \mid r x \in R d\},
$$

and so $|R / A| \leq|R / R d|=B$ for the left ideal $A=\{r \in R \mid r x \in N d\}$. This shows that each cyclic submodule of $(N \cap D) / N D$ has Krull dimension less than or equal to $\beta$, which implies that $|(N \cap D) / N D| \leq \beta<\alpha$. Thus $|N / N D|<\alpha$ since $|N / N D|=\sup \{|N /(N \cap D)|,|(N \cap D) / N D|\}$ and $|N /(N \cap D)|=|(N+D) / D| \leq|R / D|<\alpha$.

THEOREM 6'. Let $R$ be a ring with Krull dimension $\alpha$ on the left, and let $N_{\alpha}$ be the intersection of all minimal prime ideals $P$ of $R$ such that $|R / P|=\alpha$. Then the prime radical $N$ of $R$ is left weakly ideal invariant if and only if $C\left(N_{\alpha}\right)$ is a left ore set and $\tau_{\alpha}$ is a torsion radical.

Proof. If $N$ is left weakly ideal invariant, then by part (a) of the preceding proposition, $\tau_{\alpha}$ is a torsion radical. The proof of Theorem 6 of [1] then shows that $C\left(N_{\alpha}\right)$ is a left ore set, since by part (b) of the preceding proposition, $N$ is left weakly ideal invariant if and only if $N_{\alpha}$ is left weakly ideal invariant.

The converse follows from the preceding proposition and the proof of Theorem 6 of [1].

\section{References}

[1] John A. Beachy, "Weak ideal inyariance and orders in Artinian rings", Buzl. Austral. Math. Soc. 23 (1981), 255-264.

[2] K.A. Brown, T.H. Lenagan and J.T. Stafford, "Weak ideal invariance and localisation", J. London Math. Soc. (2) 21 (1980), 53-61. 
[3] Robert Gordon and J.C. Robson, Kmull dimension (Memoirs of the American Mathematical Society, 133. American Mathematical Society, Providence, Rhode Island, 1973).

[4] Gunter Krause, T.H. Lenagan and J.T. Stafford, "Ideal invariance and Artinian quotient rings", J. Algebra 55 (1978), 145-154.

Department of Mathematical Sciences, Northern Illinois University, DeKalb, IIIinois 60115, USA. 